\title{
GEOMETRY IN CERTAIN SIMPLE GROUPS
}

\author{
EICHI ABE
}

(Received August 31,1961)

1. Introduction, Certain finite groups can be considered in a natural manner as geometrical groups in the sense that they, along with a class of conjugate subgroups, characterize a certain geometry and also act as groups of motions on this geometry. G. Bachman [2] and W. Magnus [4] have defined the groups of motions in terms of properties of the group itself and studied some special class of such groups of finite order. In this note, modifying their definitions, we shall define certain groups of motions (of finite or infinite order) and study some structural properties of certain simple groups as groups of motions.

2. Basic definitions and axioms. We shall define the groups of motions in the following way:

DEFINITION. 1. A group $G$ is called a group of $n$-dimensional motions if it satisfies the following conditions :

(1) There exists a set $\sum$ of proper maximal subgroups $M_{\alpha}$ of $G$ which consists of the set of all subgroups conjugate in $G$ with one of the $M_{\alpha}$ of $\sum$ such that each $M_{\alpha}$ is its own normalizer.

(2) There exists a set $F$ of $n+2$ subgroups $M_{\alpha_{i}}(1 \leqq i \leqq n+2)$ in $\sum$ such that the intersection of the subgroups $M_{\alpha_{i}}(1 \leqq i \leqq n+2)$ consists of the unit element only, and there exists no subset of $\sum$ with elements less than $n+2$ having such property.

A set $F$ of $n+2$ subgroups $M_{\alpha_{t}}(1 \leqq i \leqq n+2)$ which satisfies (2) is called independent and a set of $d(<n+2)$ subgroups $M_{\alpha_{t}}(1 \leqq i \leqq d)$ is called also independent if, adjoining suitable subgroups $M_{\beta_{j}}(1 \leqq j \leqq n+2-d)$, we can construct an independent set $F=\left\{M_{\alpha}, M_{\beta}\right\}$. A set of subgroups in $\sum$ is called dependent if it is not independent.

The $M_{a}$ are called zero-dimensional subgroups. For $d=1,2, \ldots \ldots, n-1$, we define a $d$-dimensional subgroup as the intersection of the subgroups of an independent set with $d+1$ subgroups. The subgroup having the unit element only is called the $n$-dimensional subgroup.

We shall say that a group $G$ and a set $\sum$ satisfying (1) and (2) define an $n$-dimensional geometry in which certain postulates as specified below will be satisfied and we denote it by $\left(G, \sum\right)$. 
We shall first make some remarks on the group of $n$-dimensional motions. Suppose a group $G$ acts as a group of transformations on a point set $\sum$. Let $\Gamma$ be a subset of $\sum$. We denote by

$F(\Gamma)=$ The subgroup of $G$ leaving each point of $\Gamma$ fixed.

$F^{*}(\Gamma)=$ The subgroup of $G$ which leaves $\Gamma$ invariant (as a whole).

$\Pi(H)=$ The subset of $\sum$ left fixed (pointwise) by each element of $H$, where $H$ is a subset of elements of $G$.

The closure of a subset $\Gamma$ is defined as $\Gamma^{\prime}=F(\Gamma)$. A subset $\Gamma$ is called closed if and only if $\Gamma^{\prime}=\Gamma$. From these notations, we have the following relations :

(i) If $\Gamma_{1} \subset \Gamma_{2}$ then $F\left(\Gamma_{1}\right) \supset F\left(\Gamma_{2}\right)$ and if $H_{1} \subset H_{2}$ then $\Pi\left(H_{1}\right) \supset \Pi\left(H_{2}\right)$.

(ii) $\Gamma^{\prime \prime}=\Gamma^{\prime},\left(\Gamma_{1} \cup \Gamma_{2}\right)^{\prime} \supset \Gamma_{1}^{\prime} \cup \Gamma_{2}^{\prime}$ and $F(\Gamma)=F\left(\Gamma^{\prime}\right)$.

(iii) $F(g \Gamma)=g F(\Gamma) g^{-1} ; \Pi\left(g H g^{-1}\right)=g \Pi(H) ;(g \Gamma)^{\prime}=g \Gamma^{\prime}$ for $g \in G$.

(iv) If $\Gamma$ is a closed subset,then $N(F(\Gamma))=F^{*}(\Gamma)$, where in general $N(H)$ denote the normalizer in $G$ of $H$.

Let $M_{\alpha}=F(\alpha)$ for a point $\alpha$ of $\sum$. Suppose $N\left(M_{\alpha}\right)=M_{\alpha}$ or equivalently that a point $\alpha$ is closed in $\sum$. Since $F(g \alpha)=g M_{\alpha} g^{-1}$ for $g \in G$, if $G$ acts on $\sum$ transitively, $\sum$ may be identified with the right coset space of $G$ over a subgroup $M_{\alpha}$ on which the group $G$ acts naturally by

$$
g: x M_{x} \rightarrow g x M_{\alpha} \quad \text { for } g \in G
$$

and we have a one to one correspondence between the points of $\sum$ and the set of subgroups conjugate in $G$ with $M_{\alpha}$. (If $G$ is doubly transitive on $\sum$, then $M_{\alpha}$ is a maximal subgroup but the converse is in general not true.)

These observations lead to the following definition :

DEFINITION. 2. Let $G$ be a group which contains a complete set $\sum$ of conjugate subgroups $M_{\alpha}$ satisfying the postulation of definition 1 . Then the $M_{\alpha}$ shall be called the points of a geometry $\sum$. The group $G$ acts as a group of transformations on $\sum$ according to the rule that, for any element $g$ of $G$, the map $g\left(M_{\alpha}\right)$ of $M_{\alpha}$ is defined by

$$
g\left(M_{\alpha}\right)=g M_{\alpha} g^{-1} .
$$

For $1 \leqq d \leqq n-1$, a set $L_{d}$ of the points which are dependent to an independent set $F$ with $d+1$ points is called a $d$-dimensional subspace generated by $F$. In particular, we shall call one dimensional subspases "lines" and two dimensional subspaces "planes". $\sum$ is called an $n$-dimensional space and $n$ is called the dimension of the geometry. 
A point $M_{\alpha}$ is called incident on a subspace $L_{d}$ if $M_{\alpha}$ is a point on $L_{d}$. The action of $g$ on $L_{d}$ is defined by

$$
g\left(L_{d}\right)=g L_{d} g^{-1}
$$

Two sets $\Gamma_{1}$ and $\Gamma_{2}$ of points or subspaces are called congruent if there exists an element $g$ of $G$ such that $g\left(\Gamma_{1}\right)=\Gamma_{2}$. Now elements of $G$ are called motions of the geometry.

From the axioms (1) and (2) for $G$, we can derive the following:

(i) Any two points are congruent.

(ii) Any two $d$-dimensional subspaces are congruent if the two independent sets of points which generate the spaces are congruent.

(iii) Any $d+1$ points are independent if and only if they lie on exactly one $d$-dimensional subspace.

(iv) A motion leaving $n+2$ points fixed which are not on an $(n-1)$-dimensional subspace is the identity.

3. Simple groups of one dimensional motions. Let $G$ be the alternating group $A_{5}$ of five letters; let $\sum$ be the set of subgroups of $G$ which leave fixed one letter. Then $\left(G, \sum\right)$ define a group of one dimensional motions. More generally, let $G$ be a special projective linear group of degree 2 with coefficients in a field $K$. Let $\widetilde{G}=S L(2, K)$ and $\boldsymbol{\rho}$ be the natural homomorphism of $\widetilde{G}$ onto $G$. We set

$$
\begin{gathered}
\widetilde{B}=\left\{\left(\begin{array}{rr}
z & t \\
& z^{-1}
\end{array}\right) ; t \in K, z \in K^{*}\right\}, \varphi \widetilde{B}=B, \\
\widetilde{T}=\left\{\left(\begin{array}{ll}
z & \\
& z^{-1}
\end{array}\right) ; z \in K^{*}\right\}, \varphi \widetilde{T}=T \\
\varphi\left(\begin{array}{rr}
1 & t \\
& 1
\end{array}\right)=x(t), \varphi\left(\begin{array}{ll}
z & \\
& z^{-1}
\end{array}\right)=h(z), \varphi\left(\begin{array}{r}
1 \\
-1
\end{array}\right)=\omega,
\end{gathered}
$$

Then $G$ and the set $\sum$ of subgroups conjugate with $B$ define a group of one dimensional motions. The intersection of any two distinct subgroups in $\sum$ is a subgroup conjugate with $T$ and the intersection of any three distinct subgroups in $\sum$ is the identity. If we fix a subgroup $B_{\infty}$ in $\sum$, then other subgroups in $\sum$ can be represented by

$$
B_{t}=x(t) \omega B_{\infty} \omega^{-1} x(t)^{-1} .
$$

Moreover, we have 


$$
\begin{aligned}
h(z) B_{t} & =B_{z^{2} t}, x(u) B_{t}=B_{t+u} \\
\omega B_{0} & =B_{\infty}, \quad \omega B_{t}=B_{t^{-1}}
\end{aligned}
$$

Thus $\sum$ can be identified with a projective line $(K, \infty)$ on which $G$ acts as a group of linear fractional substitutions.

It should be noticed that $G$ acts on $\sum$ satisfying

(*) $G$ is triply transitive and no non-trivial motion leaves three points fixed.

We note that the simple groups defined by M. Suzuki [6] and R. Ree [5], which can be imbeded in the Chevalley's simple groups of types $B_{2}$ and $G_{2}$, may be interpreted as groups of one dimensional motions. We shall introduce the groups. (As for the notations of Chevalley's simple groups used in the following, see Abe [1].)

Let $K$ be a finite field with $q=2^{2^{n+1}}(n \geqq 1)$ elements ; let $\theta$ be the automorphism of $K$ defined by $\theta(\alpha)=\alpha^{2^{n}}$ for $\alpha \in K$. Now let $G$ be a Chevalley's simple group of type $B_{2}$ defined over the field $K$; let $\sigma$ be the automorphism of $G$, which satisfies $\sigma^{2}=1$, defined by

$$
x_{a}(t)^{\sigma}=x_{b}\left(t^{2 \theta}\right) ; x_{b}(t)^{\sigma}=x_{a}\left(t^{\theta}\right)
$$

where $(a, b)$ is a fundamental root system such that $a\left(H_{b}\right)=1$.

Let $G^{1}$ be the subgroup of $G$ contisting of all elements $x$ such that $x^{\sigma}=x$. Then $G^{1}$ is a simple group of order $q^{2}(q-1)\left(q^{2}+1\right)$. We set $B^{1}=\mathfrak{H} 5 \cap G^{1}$. Let $\sum$ be the set of the subgroups conjugate with $B^{1}$. Then $\left(G, \sum\right)$ defines a group of one dimensional motions.

N.B. The isomorphism of $G$ into $G L(4, K)$ defined by

$$
\psi: x_{a}(t) \rightarrow\left(\begin{array}{cccc}
1 & & & \\
& 1 & & \\
& t & 1 & \\
& & & 1
\end{array}\right) ; \quad x_{b}(t) \rightarrow\left(\begin{array}{cccc}
1 & & & \\
t & 1 & & \\
& & 1 & \\
& & t & 1
\end{array}\right)
$$

induces the isomorphism of $G^{1}$ into $G L(4, K)$. The image $\psi\left(G^{1}\right)$ is the group defined by Suzuki. For example, any element in $\mathfrak{u}^{1}=\mathfrak{U} \cap G^{1}$ can be represented by the form

$$
x(t, u)=x_{a}(t) x_{b}\left(t^{\theta}\right) x_{a+b}(u) x_{a+2 b}\left(t^{2 \theta+1}+u\right)
$$

where $t, u \in K$ and we have

$$
\psi(x(t, u))=\left(\begin{array}{llll}
1 & 1 & \\
t^{\theta} & t & 1 & \\
t^{\theta+1}+u & u & t^{\theta} & 1
\end{array}\right)
$$

Let $K$ be a finite field with $q=3^{2^{n+1}}(n \geqq 1)$ elements; let $\theta$ be the auto- 
morphism of $K$ defined by $\theta(\alpha)=\alpha^{3^{n}}$ for $\alpha \in K$. Now let $G$ be a Chevalley's simple group of type $G_{2}$ defined over the field $K$; let $\sigma$ be the automorphism of $G$, which satisfies $\sigma^{2}=1$,defined by

$$
x_{a}(t)^{\sigma}=x_{b}\left(t^{3 \theta}\right) ; x_{b}(t)^{\sigma}=x_{a}\left(t^{\theta}\right)
$$

where $(a, b)$ is a fundamental root system such that $a\left(H_{b}\right)=1$.

Let $G^{1}$ be the subgroup of $G$ consisting of all elements $x$ such that $x^{\sigma}=x$. Then $G^{1}$ is a Ree's simple group of order $q^{3}(q-1)\left(q^{3}+1\right)$. We set $B^{1}=\mathfrak{H}$ S $\cap G^{1}$; let $\sum$ be the set of subgroups of $G^{1}$ conjugate with $B^{1}$. Then $\left(G^{1}, \sum\right)$ defines a group of one dimensional motions. It should be noticed that these groups act on $\sum$ satisfying

$(* *) G$ is doubly transitive and no non-trivial motion leaves three points fixed.

The finite groups which satisfies the condition $\left(^{* *}\right)$ have been studied by many persons and it is known that these groups with some additional conditions are simple groups or Frobenius groups. We may also prove that

(**) A finite group which satisfies the condition (**) and which is its own commutator group is a simple group.

4. Chevalley's simple groups. We shall show some geometric structure of Chevalley's simple groups. We shall use the following notations :

$G$ : Chevalley's simple group.

$\Delta$ : The system of roots of the simple Lie algebra from which $G$ is constructed. $W(\Delta)$ : The Weyl group of $\Delta$.

$\left(a_{1}, \ldots \ldots, a_{n}\right):$ A fundamental root system of $\Delta$ which is fixed once for all and are numbered as in the figure 1 in [1].

$\Delta_{0}^{(i)}$ : The set of roots $r \in \Delta$ such that $r=\sum \lambda_{k} a_{k}, \lambda_{i}>0$.

$\Delta^{[i]}$ : The set of roots $r \in \Delta$ such that $r=\lambda_{i+1} a_{i+1}+\ldots \ldots+\lambda_{n} a_{n}$.

$w_{r}$ : The element of $W(\Delta)$ which is the reflexion by $r$.

$W^{[i]}$ : The subgroup of $W(\Delta)$ generated by $w_{r}, r \in \Delta^{[i]}$.

$G^{(i)}$ : The maximal subgroup of $G$ defined in [1].

$\mathfrak{U}\left(\Delta_{c}^{(i)}\right)$ : The subgroup of $G$ generated by $\mathfrak{X}_{r}, r \in \Delta_{0}^{(i)}$.

$\mathfrak{S}\left(\Delta^{[j]}\right)$ : The subgroup of $G$ generated by $\mathfrak{X}_{r}, r \in \Delta^{[i]}$.

(i) Case of type $A_{n}$.

Let $G$ be of type $A_{n} ; \sum$ be the set of the subgroups conjugate with $G^{(1)}$.

It is easy to show that $G^{(1)}$ is its own normalizer. $G$ acts on $\sum$ transitively according to the rule that, for any element $g$ of $G, g \alpha=g \alpha g^{-1}, \alpha \in \sum$.

LEMMA. 1. $W=W^{[1 !}+W^{[1]} w_{a_{1}} W^{[1]}$ 
PROOF. If $r \in \Delta_{0}^{(1)}$ and $s \in \Delta^{[1]}$, then we have $r^{\prime}=w_{s}(r) \in \Delta_{0}^{(1)}$. Thus $w_{s} w_{r}=w_{r} w_{s}$. Therefore, for any $w \in W(\Delta)$, we may represent $w$ as

$$
w=w_{r_{1}} w_{r_{2}} \cdots w_{r_{N}} w^{\prime}, r_{i} \in \Delta_{0}^{(1)}(1 \leqq i \leqq N), w^{\prime} \in W^{[1]} \text {. }
$$

Moreover we may assume that $r_{1}<r_{2}<\ldots \ldots<r_{N}$.

When $w_{r_{1}} w_{r_{2}} \ldots \ldots w_{r_{N}} \neq 1$. we shall prove that it is contained in $W^{[1]} w_{a_{1}} W^{[1]}$ by induction with respect to $N$. For any $r \in \Delta_{0}^{(1)}$, there exists an element $s \in \Delta^{[1 !}$ such that $w_{s} w_{r} w_{s}=w_{a_{1}}$. Therefore, the lemma is true for $N=1$. Suppose $N>1$ and the assertion is true for the case $<N$. Since $w_{r_{N-1}} w_{r_{N}}=$ $w_{r_{N}} w_{s}$ for some $s \in \Delta^{[1]}, w_{r_{1}} w_{r_{2}} \ldots \ldots w_{r_{N}} \in w_{r_{1}} w_{r_{2}} \ldots \ldots w_{r_{N-2}} w_{r_{N}} W^{[1]}$. Thus we may apply induction hypothesis and the lemma is proved.

LEMMA. 2. $G=G^{(1)}+G^{(1)} \omega\left(w_{a_{1}}\right) G^{(1)}$.

PROOF. Let $x \in G$, then $x$ can be represented by $x=u h \omega(w) u^{\prime}$ for $u, u^{\prime} \in \mathfrak{u}$, $h \in$ S. (cf. [3], p. 42, Théorème 2). Since $u h, u \in G^{(1)}$, we have

$$
x \in G^{(1)} \omega(w) G^{(1)} .
$$

If $w \notin W^{[1]}, x \in G^{(1)} \omega\left(w_{a_{1}}\right) G^{(1)}$ by lemma 1 .

LEMMA. 3. $G$ is doubly transitive on $\sum$.

PROOF. For $\gamma_{1}=\omega\left(w_{a_{1}}\right) \alpha_{0}, \gamma_{2}=g \alpha_{0}, g \notin \alpha_{0}$, where we denote by $\alpha_{0}$ the element $G^{(1)}$ of $\sum$, there exists an element $m \in G^{(1)}$ such that $m \gamma_{1}=\gamma_{2}$. In fact, by lemma $2, g=m \omega\left(w_{a_{1}}\right) m^{\prime}$ for some $m, m^{\prime} \in G^{(1)}$. Therefore ' $g \alpha_{0}=$ $m \omega\left(w_{a_{1}}\right) m^{\prime} \alpha_{0}=m \omega\left(w_{a_{1}}\right) \alpha_{0}$. Thus we have, for any two elements $\gamma_{1}$ and $\gamma_{2}$ of $\sum$ distinct from $\alpha_{0}$, there exists $m \in G^{(1)}$ such that $m \gamma_{1}=\gamma_{2}$.

Now let $\alpha_{1}=g_{1} \alpha_{0}, \alpha_{2}=g_{2} \alpha_{0}$ and $\beta_{1}=h_{1} \alpha_{0}, \beta_{2}=h_{2} \alpha_{0}$ be two pairs of distinct points on $\sum$. We set $\gamma_{1}=g_{1}^{-1} g_{2} \alpha_{0}, \gamma_{2}=h_{1}^{-1} h_{2} \alpha_{0}$. Since $g_{1}^{-1} g_{2}, h_{1}^{-1} h_{2} \notin G^{(1)}$, there exists an element $m \in G^{(1)}$ such that $m \gamma_{1}=\gamma_{2}$. Thus, for $g=h_{1} m g_{1}^{-1}$, we have $g \alpha_{1}=\alpha_{2}$, and the lemma is proved.

PROPOSITION. 1. The intersection of any two subgroups in $\sum$ is conjugate with

$$
M_{1}=\mathfrak{U}\left(\Delta_{0}^{(2)}\right) \mathfrak{S} \cdot \subseteq\left(\Delta^{[2]}\right) .
$$

PROOF. It is easy to show that $M_{1}=G^{(1)} \cap \omega\left(w_{a_{1}}\right) G^{(1)} \omega\left(w_{a_{1}}\right)^{-1}$. On the other hand, by lemma 3 , for any two distinct elements $\gamma_{1}, \gamma_{2}$ on $\sum$, there exists an element $g \in G$ such that $g \gamma_{1}=\alpha_{0} \quad g \gamma_{2}=\omega\left(w_{a_{1}}\right) \alpha_{0}$. Thus we have $\gamma_{1} \cap \gamma_{2}=g^{-1} \alpha_{0} \cap g^{-1} \omega\left(w_{a_{1}}\right) \alpha_{0}=g^{-1} M_{1} g$. This completes the proof.

If an element $g$ of $G$ can be represented by $x_{a_{1}}(t) \omega\left(w_{a_{1}}\right)$ for $t \in K$, the intersection of subgroups $\alpha_{0}, \omega\left(w_{a_{1}}\right) \alpha_{0}$ and $g \alpha_{0}$ contains $\mathfrak{u}\left(\Delta_{0}^{(2)}\right)$ and $\subseteq\left(\Delta^{[2]}\right)$. For 
any element $g \neq x_{a_{1}}(t) \omega\left(w_{a_{1}}\right)$ of $G$, the intersection of subgroups $\alpha_{0}, \omega\left(w_{a_{1}}\right) \alpha_{0}$ and $g \alpha_{0}$ is conjugate with

$$
M_{2}=\mathfrak{H}\left(\Delta_{0}^{(3)}\right) \cdot \mathfrak{s} \cdot \subseteq\left(\Delta^{[3]}\right) .
$$

In the same way, we may prove the following:

PROPOSITION. 2. Let $g^{\prime}=x_{r_{1}}\left(t_{1}\right) \ldots \ldots x r_{N}\left(t_{N}\right)\left(w_{r_{1}} \ldots \ldots w_{r_{N}}\right)$ where $t_{i} \in K$, $\neq 0, r_{i} \in \Delta_{0}^{(1)}(1 \leqq i \leqq N)$ and $r_{1}<r_{2}<\ldots \ldots<r_{N} \leqq s_{d-1}=a_{1}+a_{2}+\cdots \cdots+a_{d-1}$ $(d>n)$. Then the intersection of $\alpha_{0}, \omega\left(w_{a_{1}}\right) \alpha_{0}, \ldots, \omega, \omega\left(w_{s_{d-1}}\right) \alpha_{0}$ and $g^{\prime} \alpha_{0}$ contains $\mathfrak{u}\left(\Delta_{0}^{(d)}\right)$ and $\mathfrak{u}\left(\Delta^{[d]}\right)$.

For any element $g \neq g^{\prime}, \neq 1$ of $G$, the intersection of $\alpha_{0}, \omega\left(w_{s_{1}}\right) \alpha_{0}, \ldots \ldots$, $\omega\left(w_{s-1}\right) \alpha_{0}$ and $g \alpha_{0}$ is conjugate with

$$
M_{d}=\mathfrak{H}\left(\Delta_{0}^{(a+1)}\right) \cdot \mathfrak{L} \cdot \subseteq\left(\Delta^{[d+1]}\right)
$$

where $\subseteq\left(\Delta^{[n]}\right)$ is the identity.

Moreover, there exists an element $g$ of $G$ such that the intersection of $\boldsymbol{\alpha}_{0}, \omega\left(w_{s_{1}}\right) \alpha_{0}, \ldots \ldots, \omega\left(w_{s_{n}}\right) \alpha_{0}$ and $g \alpha_{0}$ is the identity,

From these observations we have the following

THEOREM. 1. Let $g$ be of type $A_{n}$ and let $\sum$ be the set of the subgroups conjugate with $G^{(1)}$, then $G$ is a group of $n$-dimensional motions. Further, $G$ satisfies the following conditions:

(3) For any $d=1,2, \ldots \ldots, n-1$, any two $d$-dimensional subgroups are conjugate in $G$.

(4) For any $d=1,2, \ldots \ldots, n-1$, if $D$ is a d-dimensional subgroup defined by an independent set $F$, then any two subgroups in $F$ are conjugate by an element of $N(D)$.

For $d=1,2, \ldots \ldots, n-1, d$-dimensional subgroups are conjugate with the subgroup

$$
M_{d}=\mathfrak{H}\left(\Delta_{0}^{(d+1)}\right) \cdot \mathfrak{S} \cdot \Im\left(\Delta^{[d+1]}\right)
$$

where $\mathfrak{S}\left(\Delta^{[n]}\right)$ is the identity. The $d$-dimensional subspaces are congruent with the set

$$
\Pi_{d}=\left\{g \alpha_{0} ; g=x_{r_{1}}\left(t_{1}\right) \ldots \ldots x_{r_{N}}\left(t_{N}\right) \omega\left(w_{r_{1}} \ldots \ldots w_{r_{N}}\right)\right\},
$$

where

$$
r_{i} \in \Delta_{0}^{(1)}, r_{1}<r_{2}<\ldots \ldots<r_{N}<s_{d}, t_{i} \in K .
$$

In particular $\Pi_{n}=\sum_{k}$. It should be noticed that $\sum$ gives the $n$-dimensional projective geometry.

(ii) Case of not type $A_{n}$. 
The geometrical structure of Chevalley's simple groups of type $A_{n}$ are somewhat different from those of type $A_{n}$. We shall show some properties of the groups.

The group $G$ of rank $n$ with the set $\sum$ of all subgroups conjugate with $G^{(1)}$ in $G$ defines a group of $n$-dimensional motions.

If $G$ is of type $B_{n}$ or $C_{n}$, it satisfies

(3') Among the one dimensional subgroups, there are two conjugate classes in $G$, For $d>1$, any two $d$-dimensional subgroups are conjugate in $G$.

(4') For any $d>1$, the $d$-dimensional subgroups satisfy the condition (4). Further, the one dimensional subgroups are conjugate with

$$
M_{1}=\mathfrak{U}\left(\Delta_{0}^{(2)}\right) \cdot \mathfrak{S} \cdot \mathfrak{S}\left(\Delta^{[2]}\right) \text { or } M_{1}^{\prime}=\mathfrak{L} \cdot \mathfrak{S}\left(\Delta^{[1]}\right)
$$

and for $d=2,3, \ldots \ldots, n-1$, the $d$-dimensional subgroups are conjugate with

$$
M_{d}=\mathfrak{U}\left(\varsigma_{0}^{(a+1)}\right) \cdot \mathfrak{5} \cdot \subseteq\left(\Delta^{[d+1]}\right) .
$$

If $G$ is of type $D_{n}(n \geqq 4)$, it satisfies

$\left(3^{\prime \prime}\right)$ For $d=1,2, \ldots \ldots, n-2$, any two $d$-dimensional subgroups are conjugate in $G$. Among the $(n-1)$-dimensional subgroups, there are two conjugate classes in $G$.

It satisfies also the condition (4).

Further, for $d=1,2, \ldots \ldots, n-3$, the $d$-dimensional subgroups are conjugate with

$$
M_{d}=\mathfrak{U}\left(\Delta_{0}^{(d+1)}\right) \cdot \mathfrak{S} \cdot S_{(}\left(\Delta^{[d+11}\right) .
$$

The $(n-2)$-dimensional subgroups are conjugate with

$$
M_{2-n}=\mathfrak{U}\left(\Delta_{0}^{(n-1)}\right) \cap \mathfrak{H}\left(\Delta_{0}^{(n)}\right)
$$

and; the $(n-1)$-dimensional subgroups are conjugate with

$$
M_{n-1}=\mathfrak{U}\left(\Delta_{0}^{(n-1)}\right) \text { or } M_{n-1}^{\prime}=\mathfrak{U}\left(\Delta_{0}^{(n)}\right) \text {. }
$$

These properties can be proved by the properties of the root system of the group in the same way as the case of type $A_{n}$. We have also the corresponding results on the groups of exceptional types. It is to be hoped that the groups of each type would be characterized by the geometrical properties.

\section{REFERECES}

[1] E. ABE, Groupes simples de Chevalley, Tôhoku Math. J., 13(1961), 253-267.

[2] G. BACHMAN, Geometry in certain finite groups, Math. Zeit. 70(1959), 466-479.

[3] C. Chevalley, Sur certains groupes simples, Tohoku Math, J., 7(1955), 14-66.

[4] W. Magnus, Some finite groups with geometrical properties, Proc. Symposium Pure Math. 1, Finite groups (1959). 
[5] R. REE, A familly of simple groups associated with simple Lie algebra of type $G_{2}$, Bull. Amer. Math. Soc., 66(1960), 508-510.

[6] M.SuZUKI, A new type of simple groups of finite order, Proc. Nat. Acad. U.S.A. 46(1960), 868-870.

Fukushima medical College. 